\title{
3D-FIB characterization of wear in WC-Co coated composites
} \section{José García ${ }^{1, a^{*}}$, Thais Carvalho Miranda ${ }^{2, b}$, Haroldo C. Pinto ${ }^{2, c}$,} Flavio Soldera ${ }^{3, d}$ and Frank Mücklich ${ }^{3, e}$

\author{
${ }^{1}$ AB Sandvik Coromant R\&D, Lerkrogsvägen 19, SE-12680, Stockholm, Sweden \\ ${ }^{2}$ Escola de Engenharia de São Carlos, Univ. de São Paulo, CEP 13566-590, Sao Carlos, Brazil \\ ${ }^{3}$ Universität des Saarlandes, Functional Materials Group, D-66123 Saarbruecken, Germany \\ ajose.garcia@sandvik.com, ${ }^{\mathrm{b}}$ thais.helena.miranda@usp.br, ${ }^{\mathrm{c}}$ haroldo@sc.usp.br, \\ d.soldera@matsci.uni-sb.de, ${ }^{\mathrm{e}}$ muecke@matsci.uni-sb.de \\ * corresponding author
}

Keywords: 3D tomography, WC-Co, wear, thermo-mechanical cracks

\begin{abstract}
In this work 3D visualization of wear in milling inserts has been investigated by Focused Ion Beam tomography. It has been observed that the morphology of the cracks differs in the z-axis direction, allowing particular characteristics of the microstructure and wear evolution to be visible. Two types of cracks develop: principal and lateral cracks. The formation of lateral cracks is strongly influenced by the degradation of the binder phase in the regions surrounding the principal crack. The lateral cracks and the deflection of the main cracks present a particular semi-elliptical geometry, which correlates with the stress field originated during the input of a cycling load in a fatigue condition.
\end{abstract}

\section{Introduction}

Cemented carbides made of WC-Co coated with wear resistant layers are the first option for machining of metallic alloys in the automotive and aerospace industry [1]. The productivity of the machining process is linked to the wear resistance of the composite material. Depending on application different types of wear mechanisms, such as flank and crater wear, plastic deformation or comb cracks are the limiting factor for performance. Comb cracks are the main wear mechanism in inserts for milling applications [2]. Comb cracks form during thermo-mechanical cycling loading [3]. A challenge is the characterization of the wear, which defines the design of the cutting tool material to withstand the machining conditions. In many cases 2D information does not reveal the details that are necessary to understand how wear evolves, which may lead to incomplete interpretation of the wear process, since forces, heat input and deformation in the cutting edge are in $3 \mathrm{D}$ conditions.

The main objective of this work is to carry out the three-dimensional characterization of comb cracks. The 3D characterization is carried out using Focused Ion Beam (FIB) tomography. The technique consists of serial sectioning of the microstructural area of interest using a Focused Ion Beam. Basically, a Gallium ion source is used to remove the material and an electron beam to capture the images slice by slice. Further reconstruction of the serial cuts using commercial software leads to the 3D image [4].

\section{Experimental}

A cutting insert made of cemented carbide coated with a multilayer applied by chemical vapour deposition (CVD) was investigated. The chemical composition of the cemented carbide was WC-6.3Co-0.63Cr. The coating consists of a TiN bonding layer to the substrate of ca. $1 \mu \mathrm{m}$, followed by a MT-Ti(C,N) layer of 3-4 $\mu \mathrm{m}$ and an $\alpha-\mathrm{Al}_{2} \mathrm{O}_{3}$ layer is 3-4 $\mu \mathrm{m}$ and a TiN-layer, which is top-blasted on the insert's surface. 
Comb cracks were generated by wet milling tests in grey cast iron. Using this method the inserts are subjected to a high thermo-mechanical cycling condition due to the use of coolant, which causes the formation of comb cracks perpendicular to the cutting edge. In this test the tool life is mostly determined by chipping as a consequence of the comb cracks. So to avoid chipping and make the comb crack visible the test was stopped at $25 \%$ of the total tool life (Fig.1.).

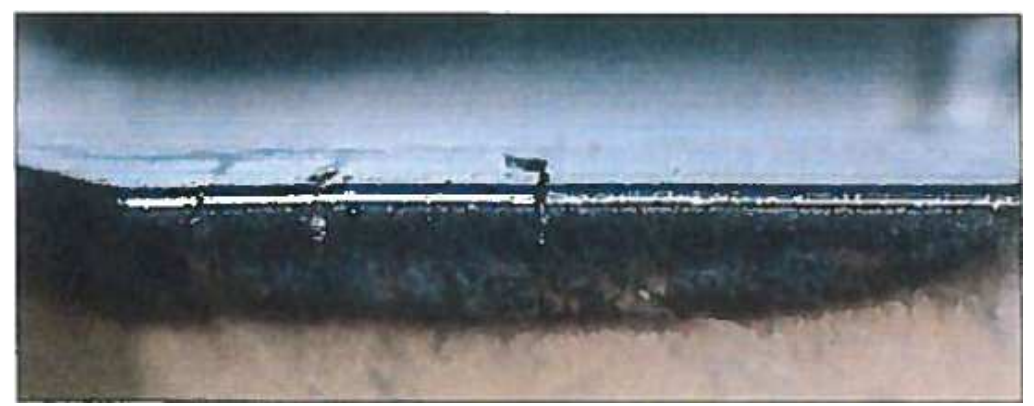

Figure 1: Stereographic image of the insert edge after the milling test. 3 comb cracks are clearly visible.

\section{Results and discussions}

\section{Method development}

A method for FIB tomography was developed using two dual beam systems Strata DB235 and Helios 600 (FEI Company) at University of Saarlandes (Functional Materials Group). The instruments incorporate both a FIB and a field emission scanning electron microscope (FE-SEM).

The whole insert was covered with a conductive layer by sputtering gold to avoid surface charging. The sample was attached to the sample holder with adhesive copper tape and a drop of colloidal graphite. Before starting the milling procedure it was necessary to deposit a platinum layer on the region to be investigated (comb crack area in this case) to protect it from the ion beam. This deposition was done in-situ by ion beam induced deposition (IBID). The voltage used was $30 \mathrm{kV}$ and the current (in pA) around 10 times the area of interest (in $\mu \mathrm{m}$ ). The thickness of the layer was ca. $1 \mu \mathrm{m}$.

Then the material around the crack was removed to have the XY plane accessible for images acquisition with the electron beam to avoid shadowing effects and preventing that the re-deposited material from the milling process hinders the imaging of the cross sections. The material was removed using an ion beam with $30 \mathrm{kV}$ voltage and $22 \mathrm{nA}$ current at the Strata DB235. The last step in the preparation of the sample was the capture of the images using SEM ( $5 \mathrm{kV}$ voltage, $0.34 \mathrm{nA}$ current, EDT detector), which was done at the Helios 600.

In Fig. 2 an image of the comb crack region after some sectioning in the FIB is shown. The material removal during the serial sectioning was stopped after realizing that no change in the microstructure was taking place. The total amount of images was 400.319 sections were used to make the reconstruction. 


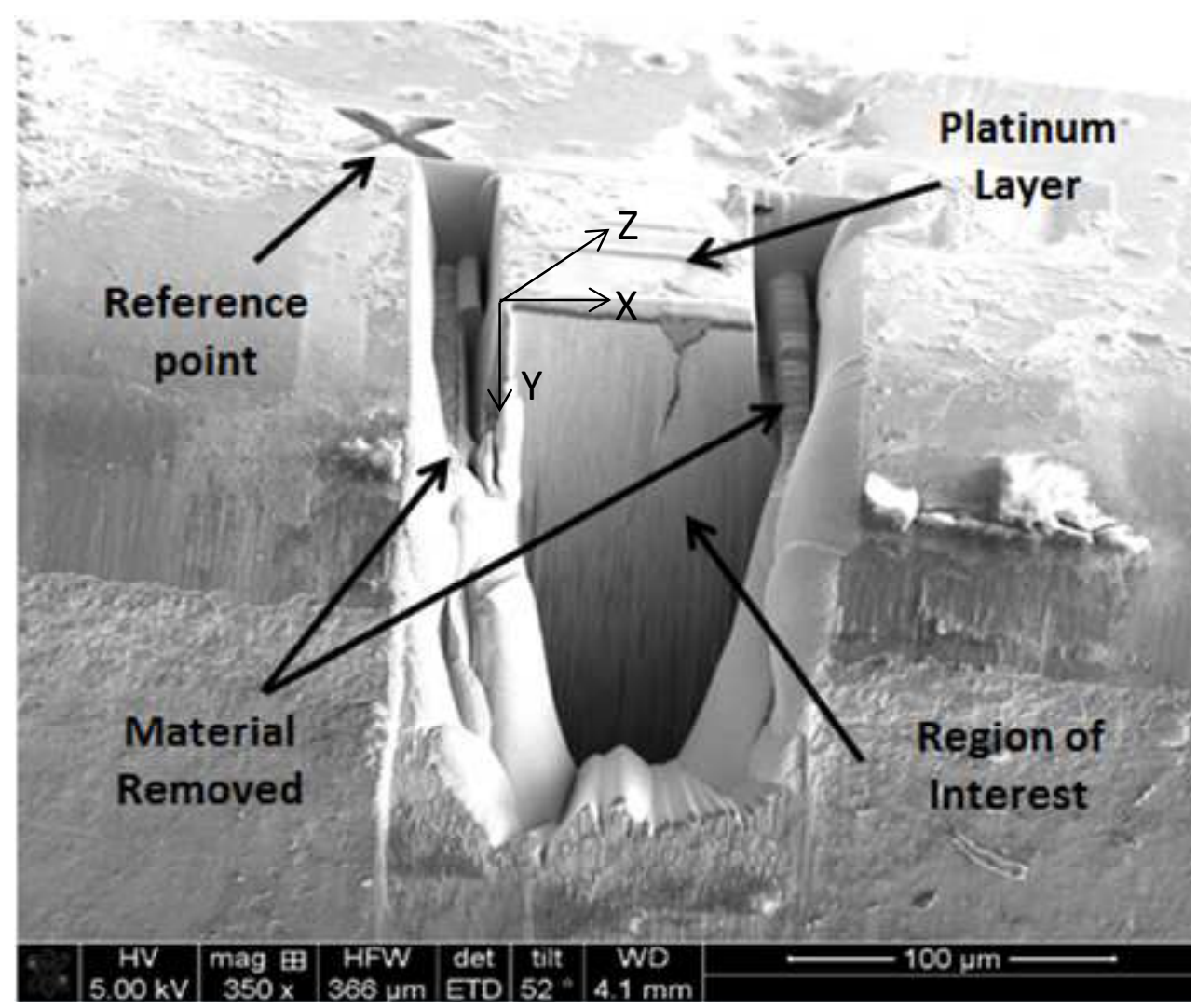

Figure 2: SEM image of the sample during the FIB tomography.

\section{Serial sectioning and $3 D$ reconstruction}

Figure 3 shows a series of images taken by the SEM (slide 18, 50, 137, 200 and 318) which were used for the $3 \mathrm{D}$ reconstruction. The images show the microstructure of the region around the comb crack at different distances from the flank surface (z-axis).

These images provide valuable information which is not possible to observe from a single 2D image. In particular the microstructural changes connected to the region around the crack are visible. For example the amount of workpiece material micro-welded to the cutting material varies inside the crack. Also the morphology of the wear track on the crater surface changes in the zdirection. Most interestingly it is observed that other cracks parallel to the edge line are visible. These lateral cracks are inside of the material as shown in slides 50, 137, 200 and 318. It can be observed that the microstructure around the lateral crack region is different compared to the initial microstructure of the material not affected by the wear. The distance of these lateral cracks varies as function of depth (z-axis) which may be assumed as independent cracks; however the 3D reconstruction will allow us to observe that this variation of depth to the edge line surface of the lateral cracks is connected to a particular morphology of them.

All the 319 images were opened together in the commercial AMIRA software and worked out (aligned and segmented) to obtain the $3(\mathrm{X}, \mathrm{Y}, \mathrm{Z})$ dimensional visualization of the full comb crack and its surroundings. The result is shown in Fig. 4.

From the reconstruction it is observed that, by connecting the lateral cracks images in the $3 \mathrm{D}$ reconstruction, they present a particular morphology. The lateral crack has a semispherical morphology, which connects to the crater surface of the insert. This morphology can be more visible if the $3 \mathrm{D}$ reconstruction of the lateral crack is intercepted by a dark plane, as shown in Fig. 5. The lateral cracks present this particular form due to the fact that they are formed by the cycling mechanical load (fatigue condition) during the milling process. 


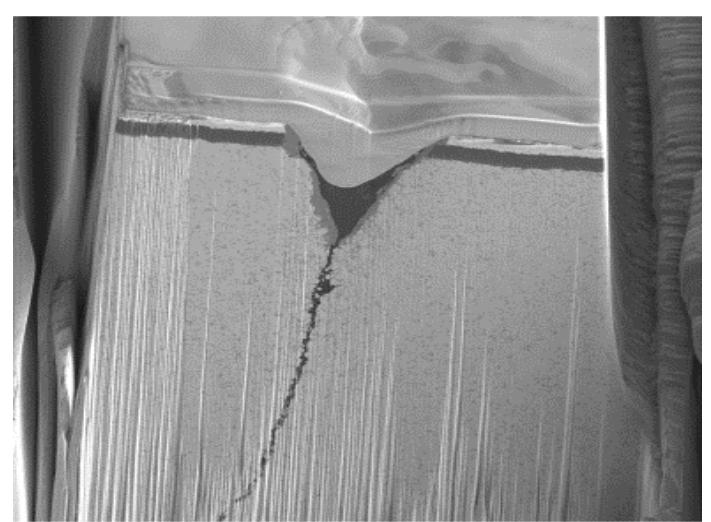

18

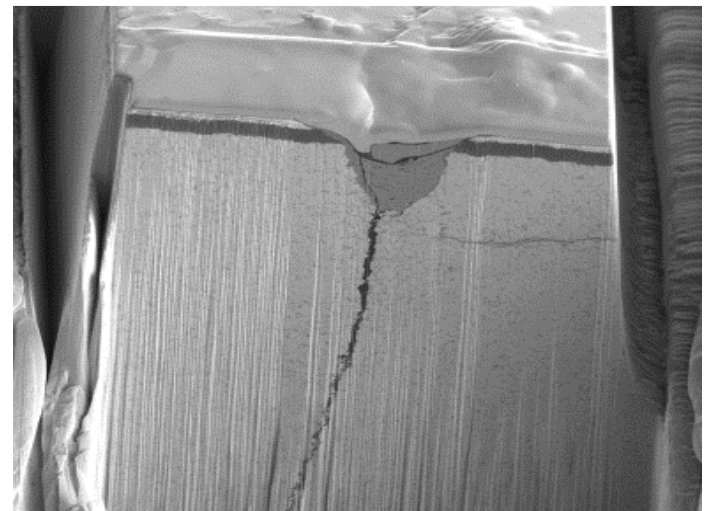

137

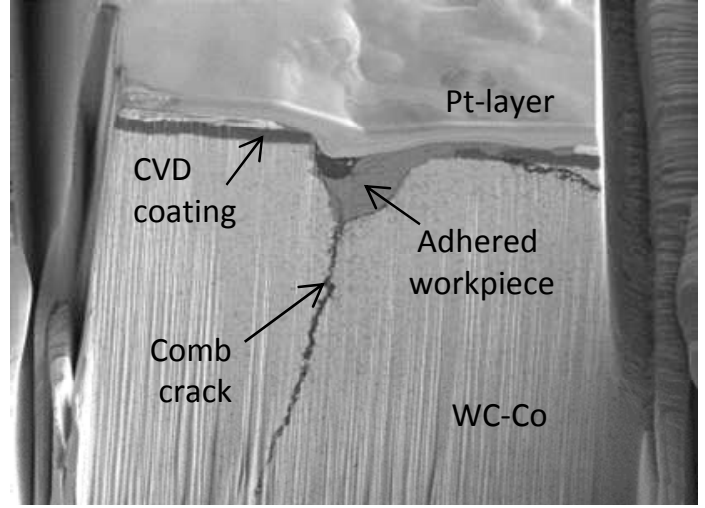

50

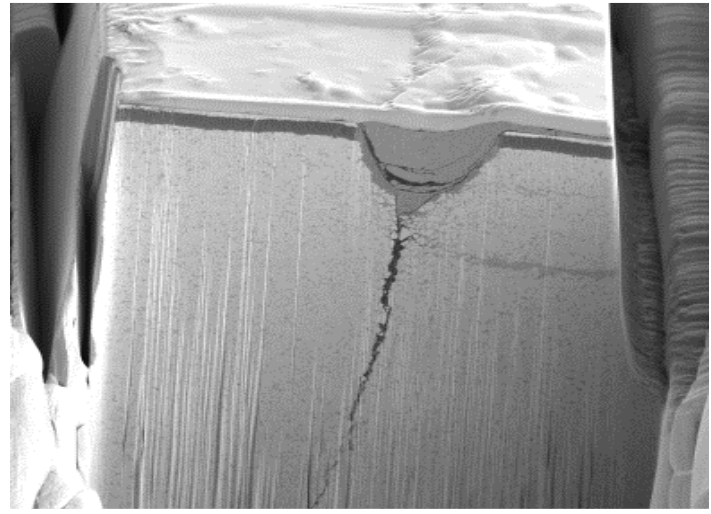

200

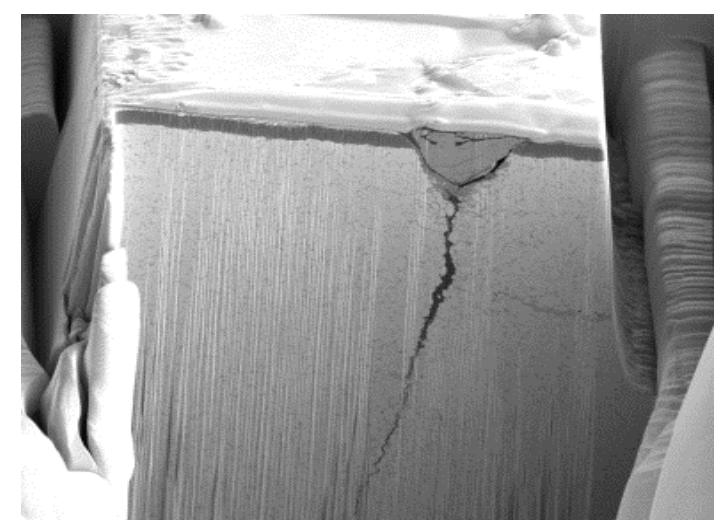

318

Figure 3: SEM images used for the reconstruction, showing microstructure changes at the different places of the region of interest.

This is typical for brittle materials under cycling (increase/decrease) load condition, as demonstrated by Hutching [5]. It is remarkable that this particular form seems to be connected to the stress field acting in the cutting edge during the machining process, since the stress distribution of forces has the same semispherical form as the one observed in these lateral cracks. This correlation between stress and crack formation has been modelled by Sergejev et al in WC-Co cemented carbides [6]. They carried out theoretical calculations on the geometry of fatigue cracks on cemented carbides developed from cyclical loading (indentation cracks for different indentation loads); and the connection to lateral crack formation. It was determined that the number of lateral cracks that would be observed from one cross section of cemented carbide was dependent on the loading force and the number of loading cycles. The propagation of the primary lateral cracks was considered as giving way for surface fatigue fragments formation. The lateral cracks were determined to propagate with an elliptical form towards the free surface of the material. 


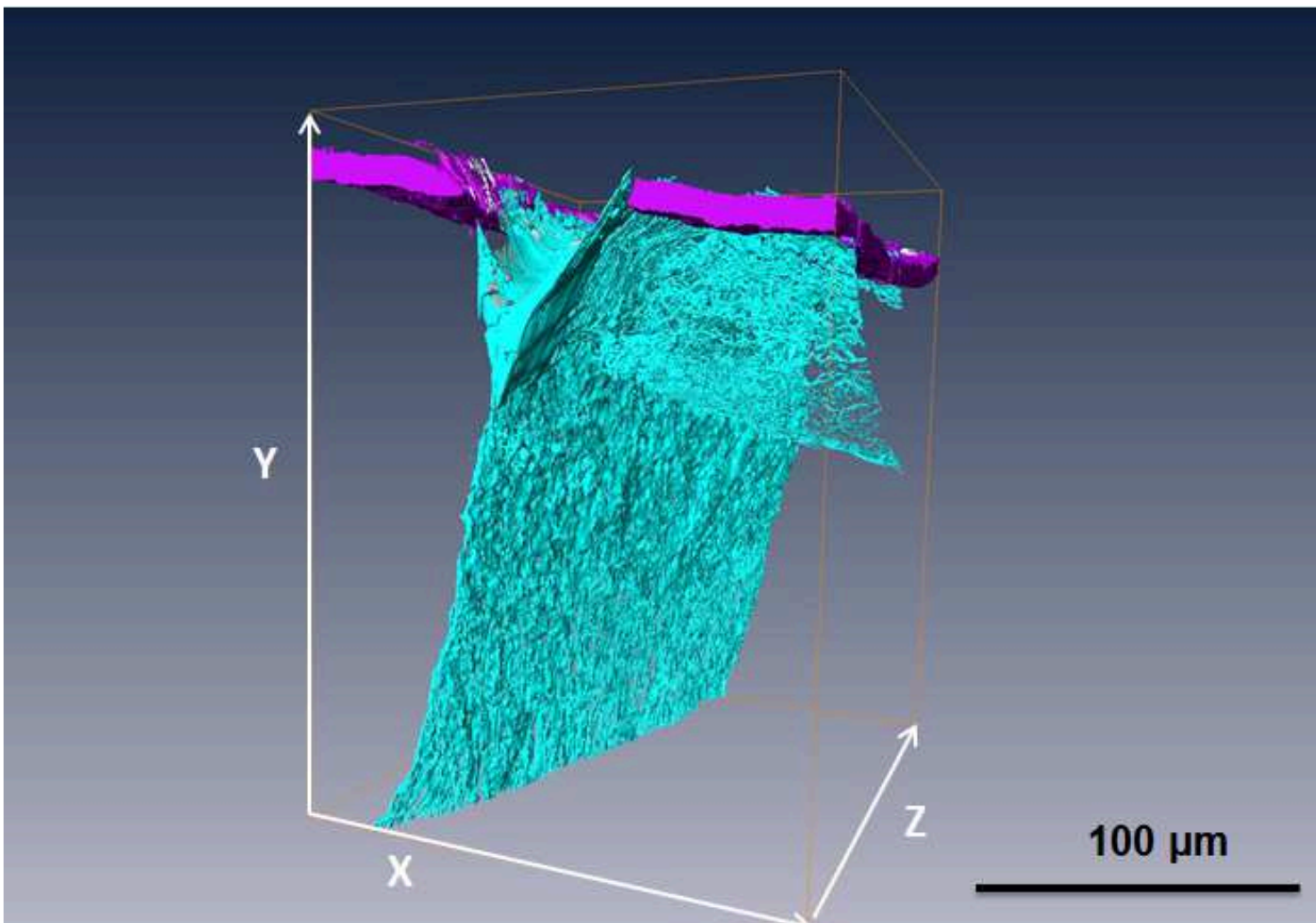

Figure 4: $3 \mathrm{D}$ visualization of the comb crack investigated. The crack is marked with Cyan and the coating is marked with Violet.

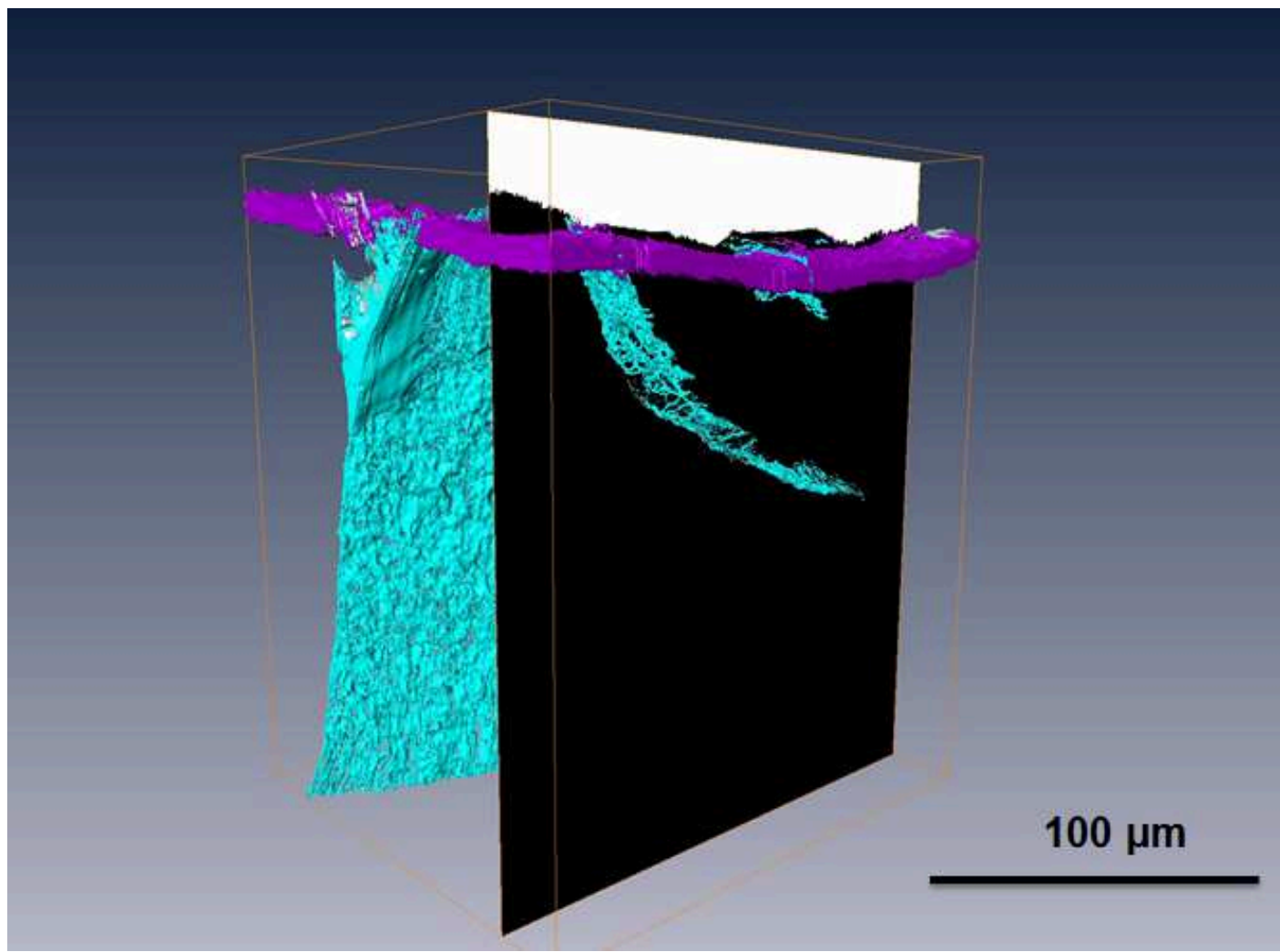

Figure 5: The principal crack with the lateral crack, parallel from the surface. A black plane has been drawn in the $y-z$ plane in order to visualize the morphology of the lateral crack. 
Furthermore the formation of lateral cracks may be connected to microstructural changes in the region surrounding the crack, such as depletion of binder by chemical attack of the cooling media, as shown by the 3D sectioning in Fig. 3. The propagation of cracks may be dominated by the interfaces WC-to-Co, which depends on the particle size, distribution of the WC grains but also on the binder composition and the interfacial energy of WC/WC and $\mathrm{WC} / \mathrm{Co}$ interfaces [7].

\section{Conclusions}

A method for 3D-FIB tomography of wear has been developed and applied successfully to $3 \mathrm{D}$ visualization of the main wear in milling inserts, the comb cracks. It has been observed that the morphology of the cracks may differ in the z-axis direction, allowing other characteristics of the microstructure and wear to be visible. In particular the formation and morphology of the principal crack and the lateral cracks connected to the principal comb crack was observed, which is linked to microstructural degradation in the surroundings of the crack. It can be concluded that 3Dreconstruction can be applied to investigate the wear of cutting inserts and to further understand the link between microstructure and performance of the tools.

\section{Acknowledgements}

The authors would like to thank the support of the EU Commission through the RISE project Create-Network (Project No 644013). F. Mücklich and F. Soldera thank the EFRE Funds of the EU Commission within the AME-Lab project.

\section{References}

[1] J. García, On the formation of graded microstructures of Fe-Ni-Co based cemented carbides and CVD Ti-C-N coatings for cutting tools. Habilitationsschrift, Technische Universität Wien, Austria, 2013.

[2] Modern Metal Cutting. A practical handbook. Sandvik Coromant R\&D, 1994.

[3] A.C.A de Melo, Estudo das trincas de origem térmica geradas em ferramentas de metal duro durante o fresamento frontal. PhD thesis, Univ. Fed. de Uberlandia, Brazil, 2001.

[4] F. Soldera, F. Lasagni and F. Mücklich, Nano Characterization of Structures by Focused Ion Beam (FIB) Tomography. Fabrication and Characterization in the Micro-Nano Range Advanced Structured Materials, Berlin, Germany, 2011, Volume 10, pp. 171-199.

[5] I.M. Hutchings, Tribology: Friction and Wear of Engineering Materials. 2001, pp. 150-155.

[6] F. Sergejev, I. Preis, J. Kübarsepp and M. Antonov, Correlation between surface fatigue and microstructural defects of cemented carbides, Wear 264, 2007, 770-774.

[7] K. Holmberg, A. Laukkanen, A. Ghabchi, M. Rombouts, E. Turunen, R. Waudby, T.Suhonen, K. Valtonen and E. Sarlin, Computational modeling based wear resistance analysis of thick composite coatings, Tribology Int. 72, 2013, 13-30. 